\section{P12.11 REACH OF STI CLINICS BY HIGH-RISK ETHNIC GROUPS IN THE NETHERLANDS}

${ }^{1} \mathrm{AAM}$ van Oeffelen, ${ }^{1,2,3} \mathrm{HM}$ Götz*${ }^{4}{ }^{4} \mathrm{~J}$ Van Steenbergen, ${ }^{5} \mathrm{FAM}$ van Leeuwen-Voerman, ${ }^{6} \mathrm{AMC}$ van Lier, ${ }^{7} \mathrm{MG}$ van Veen, ${ }^{1} \mathrm{BHB}$ van Benthem, 'IVF van den Broek. ${ }^{1}$ Department of Epidemiology and Surveillance, National Institute for Public Health and the Environment, Bilthoven, The Netherlands; '2Public Health Service Rotterdam- Rijnmond, Rotterdam, The Netherlands; ${ }^{3}$ Department of Public Health, Erasmus MC, University Medical Center Rotterdam, The Netherlands; ${ }^{4}$ Academic Center LUMC, Leiden. the Netherlands; ${ }^{5}$ Public Health Services Haaglanden, The Hague, The Netherlands; ${ }^{6}$ Public Health Service Region Utrecht, The Netherlands; ${ }^{7}$ Public Health Service Amsterdam, Department Infectious Diseases, Amsterdam, The Netherlands

\subsection{6/sextrans-2015-052270.49}

Background In the Netherlands, ethnic minorities from STI endemic countries are considered to be a high-risk group qualifying for STI clinic care free of charge. It is unknown to what extent they reach STI clinics.

Objectives To investigate the difference in STI clinic consultation- and positivity rate between ethnic minority groups and ethnic Dutch, and compare findings between the 4 largest cities.

Methods Between 2011 and 2013, all STI clinic consultations in Amsterdam, Rotterdam, The Hague and Utrecht of persons aged 15 to 44 years in their place of living were selected from the national STI surveillance database. These were combined with numbers of inhabitants per ethnic group (in the same age range) to calculate consultation rate as a proxy for coverage of the STI clinics in each city. Negative binomial regression (adjusted for age and sex) was used to compare the STI consultation- and positivity rate between ethnic Dutch and ethnic minorities, and between ethnic groups living in Amsterdam, Rotterdam, The Hague and Utrecht.

Results Ethnic minorities from Eastern Europe, Sub-Saharan Africa, Suriname, Netherlands Antilles/Aruba and Latin America have a higher consultation rate (range RR 1.26-2.21) than ethnic Dutch, whereas ethnic minorities from other European countries, Turkey, North Africa and Asia have a lower consultation rate (range RR 0.29-0.83). All ethnic minority groups have a higher positivity rate than ethnic Dutch (range RR 1.22-1.71). When comparing cities, among all ethnic groups consultation rate is markedly highest in Amsterdam, and lowest in Utrecht (RR Amsterdam vs. Utrecht (range): 4.30-10.71). Positivity rate is more similar between cities.

Conclusion There are substantial differences in the coverage of ethnic minorities by metropolitan STI clinics in the Netherlands. Although high positivity rates among ethnic minorities suggests that persons at higher risk reach STI clinics, focusing on ethnic groups with low consultation rates remains important.

Disclosure of interest statement No grants were received in the development of this study.

\section{P12.12 BACTERIA AETIOLOGY OF SEXUALLY TRANSMITTED INFECTIONS AT SEXUALLY TRANSMITTED INFECTION CLINIC IN KUMASI, GHANA; USE OF MULTIPLEX REAL TIME POLYMERASE CHAIN REACTION}

\footnotetext{
${ }^{1}$ Augustina Angelina Sylverken, ${ }^{1,2}$ Ellis Owusu-Dabo*, ${ }^{1}$ Denis Dekugmen Yar, ${ }^{1,3}$ Samson Pandam Salifu, ${ }^{1}$ Nana Yaa Awua-Boateng, ${ }^{1}$ John Humphrey Amuasi, ${ }^{1}$ Portia Boakye Okyere, ${ }^{1,2}$ Thomas Agyarko-Poku*. 'Kumasi Centre for Collaborative Research in Tropical Medicine, Kwame Nkrumah University of Science and Technology (KNUST), Kumasi, Ghana; ${ }^{2}$ School of Public Health, KNUST, Kumasi, Ghana; ${ }^{3}$ Department of Biochemistry and Biotechnology, KNUST, Kumasi, Ghana; ${ }^{4}$ Suntreso Government Hospital, Ghana Health Services, Kumasi, Ghana
}

10.1136/sextrans-2015-052270.492
Introduction Most sexually transmitted infection (STI) management efforts focus on the syndrome approach to diagnose and treat patients who are symptomatic. However, most women with STIs are known to be entirely asymptomatic or if symptoms exist are often unrecognised either clinically or bacteriologically. We determine the true prevalence and aetiology of STIs among these women in Ghana.

Methods This cross sectional study conducted between February and April 2014 employed a sensitive multiplex real time Polymerase chain reaction (PCR) assay that simultaneously detects seven STI bacteria pathogens in 200 women attending a Specialist STI clinic at Suntreso Government Hospital in Kumasi, Ghana. Data was captured and analysed using Stata version 12.

Results A total $156(78.00 \%)$ of the women were asymptomatic. Overall, $175(87.5 \%)$ of the women tested positive for at least one bacteria STI pathogen of which $50(25.0 \%)$ had single infections, $76(38.0 \%)$ and 39 (19.5\%) had double and triple infections respectively. Mycoplasma hominis was the most commonly detectable pathogen present in $135(67.5 \%)$ women. Altogether, $135(86.54 \%)$ and $40(90.91 \%)$ of the symptomatic and asymptomatic women tested positive for at least one pathogen $(\mathrm{p}<$ $0.05)$. There were no significant associations $(\mathrm{p}<0.05)$ between the clinical manifestations as presented by the symptomatic women and the pathogens detected in their samples.

Conclusion Our study confirmed the importance of complementing the syndromic approach with pathogen detection and most importantly recognise that STIs in women are asymptomatic and regular empirical testing even for both symptomatic and asymptomatic patients is.

Disclosure of interest statement No conflict of interest.

\section{P12.13 INTEGRATING SEXUALLY TRANSMITTED INFECTIONS (STI) INTERVENTION PROJECT FOR FEMALE SEX WORKERS (FSW) INTO PRIMARY LEVEL OF CARE IN GHANA: ASSESSING THE IMPACT A DECADE AFTER PROJECT IMPLEMENTATION}

${ }^{1,2} \mathrm{~T}$ Agyarko-Poku* ${ }^{3} \mathrm{~N}$ Khonde. ${ }^{1}$ Department of Global and International Health, School of Public Health, Kwame Nkrumah University of Science and \& Technology, Kumasi, Ghana; ${ }^{2}$ Suntreso Government Hospital, Ghana Health Service, Kumasi, Ghana; ${ }^{3}$ West Africa Regional Disease Surveillance Capacity Strengthening Project, Accra, Ghana

\subsection{6/sextrans-2015-052270.493}

Introduction Ghana benefited from the Canadian International Development Agency funded STI project for FSW involving 9 West African countries between 1996 and 2006. The project was geared towards ensuring strong community mobilisation coupled with the integration of STI prevention, treatment, care and support into the primary level of care with the view of promoting higher coverage, local ownership and ensuring sustainability. We determine the impact of the intervention a decade after the end of the project.

Methods This descriptive cross-sectional study assessed all the 21 former project clinics from 10 administrative regions in Ghana from September $20^{\text {th }}$ to October $20^{\text {th }}, 2014$. Data was collected through site visitation, key informants interviews using structured questionnaire, survey of clinical staff and documentary review. Areas assessed were ownership, coverage, clinical and community activities and patronage.

Results 19 out of the 21 clinics were found to be operational and fully part of the Ghana Health Services health delivery system. 12 out of the 19 have been upgraded into chronic care clinics for the provision of services to patients reporting with 
conditions such as HIV/AIDS, tuberculosis, diabetes, hypertension, family planning and asthma. Available records indicated that FSW still patronised 14 of these clinics. Overall, there has been marginal increase in the FSW attendance compared with statistics during the time of the project.

Conclusion The integration of the STI intervention project into the primary level of care in the Ghana Health Services is a success. FSW like other clients who patronise health facilities deserve equal dignity, respect and quality care.

Disclosure of interest statement No conflict of interest.

\section{P12.14 CLINICAL DESCRIPTIONS OF PCR POSITIVE EARLY SYPHILIS INFECTIONS}

${ }^{1} \mathrm{JM}$ Towns*, ${ }^{2} \mathrm{DE}$ Leslie, ${ }^{1}$ I Denham, ${ }^{2} \mathrm{~F}$ Azzato, ${ }^{1,3} \mathrm{CK}$ Fairley, ${ }^{1,3} \mathrm{MY}$ Chen. ${ }^{1}$ Melbourne Sexual Health Centre (MSHC), Alfred Health; ${ }^{2}$ Victorian Infectious Diseases Reference Laboratory (VIDRL), Doherty Institute; ${ }^{3}$ Central Clinical School, Monash University

\subsection{6/sextrans-2015-052270.494}

Background Globally, syphilis remains a major and resurgent public health problem with high incidence rates in many settings, including among men who have sex with men (MSM). Left untreated, syphilis can lead to further transmission, morbidity and enhanced Human Immunodeficiency Virus (HIV) transmission. A primary chancre is classically described as an indurated single painless ulcer at the site of Treponema pallidum inoculation. However, recent clinical experience is that primary syphilis can present atypically, as multiple and/or painful ulcers with features suggestive of genital herpes. We aimed to describe serology and Treponema pallidum polymerase chain reaction (Tp PCR) positive lesions of primary syphilis in men, the rates of painful or multiple lesions, whether there was concurrent genital Herpes simplex virus (HSV) infection and whether concurrent HIV infection altered the presentation.

Methods Tp PCR positive results with confirmatory syphilis serology and HSV PCR results reported by VIDRL were identified and compared to MSHC medical records over a five-year period from 2010 to 2014.

Results 183 patients fulfilled the criteria of Tp PCR positive primary syphilis. Primary syphilis lesions were frequently painful $(49.2 \%)$ or multiple $(37.7 \%)$, and were infrequently associated with HSV (2.7\%). Presentation was not significantly altered by HIV status. Anal lesions were more common in HIV positive men $(34.2 \%)$ than in HIV negative men (11.6\%). Syphilis reinfections were more common in HIV positive men (39.5\%) than in HIV negative men (11.7\%).

Conclusion Tp PCR is a useful tool to confirm syphilis as a cause of genital lesions and positivity may precede serological markers. Early syphilis lesions may be clinically misidentified as HSV infection if syphilis is not considered. Awareness of the clinical variability of primary syphilis lesions should be included in health promotion messages to the public and health care providers.

Disclosure statement There are no conflicts of interest.

\section{P12.15 PENILE PAPULONECROTIC TUBERCULOSIS: IS IT A SEXUALLY TRANSMITTED DISEASE?}

Gupta Somesh*, R Bhatia. Department of Dermatology \& Venereology, All India Institute of Medical Sciences, New Delhi, India

10.1136/sextrans-2015-052270.495
Introduction Ulcertaive lesions of tuberculosis on penis have been classified as papulonecrotic tuberculids, though clinical presentation and absence of generalised involvement suggest that it is sexually transmitted inoculation tuberculosis.

Methods All patients with penile papulonecrotic tuberculosis (PPNT) presented during past 5 years and their wives were investigated for pulmonary and extra-pulmonary tuberculosis including reproductive tract tuberculosis (RTB). All histopathologically diagnosed patients were treated with anti-tubercular therapy (ATT) and followed up.

Results Seven patients, aged between 21-30 years, all married, were diagnosed with PPNT based on caseating epithelioid cell granulomas in histopathology. They presented with recurrent genital ulcers for the duration ranging from 3-8 years. These would start as asymptomatic papulopustules over the glans penis/prepuce which would breakdown to form painful ulcers in 1-2 weeks and heal with scarring in another 2-3 weeks. Five patients were diagnosed in the past as genital herpes due to episodic nature and received suppressive antiviral therapy without any response. All the patients denied any premarital or extra marital sexual contact or oral insertive sex. A dramatic response was seen in PPNT lesions within 4-8 weeks of starting ATT.

There was a history of primary infertility due to RTB in the spouses of two patients. Spouse of one patient conceived after a course of ATT.

Mantoux test was strongly positive in all, and PCR for Mycobacterium Tuberculosis was positive in 3 patients. HIV serology and VDRL were negative. No internal focus of tuberculosis was found.

Conclusion Several features suggest that penile tuberculide may actually be sexually acquired implantation tuberculosis. The PPNT is an isolated disease without involvement of other parts. The concurrent RTB in the spouse raises the possibility of it being sexually transmitted. With increasing acceptability of oral sex, the inoculation may take place from sputum of a patient with pulmonary TB. This hypothesis needs genotyping studies to confirm.

Disclosure of interest statement None.

\section{P12.16 OLDER PATIENTS ATTENDING SEXUALLY TRANSMITTED INFECTIONS CLINICS}

${ }^{1}$ SA Tuddenham*, ${ }^{1} \mathrm{KR}$ Page, ${ }^{2} \mathrm{CP}$ Chaulk, ${ }^{1} \mathrm{KG}$ Ghanem. ${ }^{1}$ Johns Hopkins University School of Medicine; ${ }^{2}$ Baltimore City Health Department, Baltimore, MD, USA

\subsection{6/sextrans-2015-052270.496}

Background Many older patients remain sexually active well into their eighth decade of life. Surveillance data suggest that rates of sexually transmitted infections (STIs) in this age group may be increasing. We sought to compare demographics, risk behaviours and predictors of acute infections in patients 50 years and older versus younger patients attending STI clinics in Baltimore, Maryland.

Methods Retrospective study from a large electronic database of all visits to two urban STI clinics between 2005 and 2010. Proportions were compared using the $c^{2}$ test. Logistic regression was used to assess predictors of acute STIs in older versus younger groups.

Results 4461 first visits for patients over 50 and 4893 visits for patients under 50 were included in the analysis. Patients over 50 frequently reported high-risk behaviours $[35.4 \%$ (CI $0.34-0.37$ ) vs. $52.7 \%$ (CI $0.51-0.54$ ) in those $<50$ ] but they were more 